\title{
Qualidades Psicométricas do Eating Attitudes Test (EAT-26) para Adolescentes Brasileiros do Sexo Masculino
}

\author{
Leonardo de Sousa Fortes ${ }^{1}$ \\ Universidade Federal de Pernambuco \\ Ana Carolina Soares Amaral \\ Instituto Federal de Educação de Minas Gerais \\ Sebastião de Sousa Almeida \\ Universidade de São Paulo, Ribeirão Preto/SP \\ Maria Aparecida Conti \\ Universidade Cruzeiro do Sul \\ Maria Elisa Caputo Ferreira \\ Universidade Federal de Juiz de Fora
}

\begin{abstract}
RESUMO - A pesquisa objetivou avaliar as qualidades psicométricas do Eating Attitudes Test (EAT-26) para adolescentes brasileiros do sexo masculino. A amostra foi composta por 357 jovens, com idade entre 10 e 19 anos. Os resultados evidenciaram que $32,8 \%$ variância dos dados foram explicados por um único fator e o instrumento foi capaz de diferenciar seus escores em função do estado nutricional $(p<0,05)$, além de correlacionar-se com as medidas de insatisfação corporal $(r h o=0,50)$. Confirmou sua consistência interna $(\alpha>0,88)$, não registrando diferença entre os escores (teste-reteste) $(p<0,68)$ e apresentando o coeficiente de correlação intra-classe de 0,93 . Concluiu-se que o EAT-26 comprovou suas qualidades psicométricas para o grupo estudado.
\end{abstract}

Palavras-chave: validade, reprodutibilidade, psicometria, transtornos alimentares, adolescentes

\section{Psychometric qualities of the Eating Attitudes Test (EAT-26) for Brazilian Male Adolescents}

\begin{abstract}
This research aimed to evaluate the psychometric properties of the Eating Attitudes Test (EAT-26) for male Brazilian adolescents. The sample consisted of 357 young people, aged between 10 and 19 years. The results showed that $32.8 \%$ of the data variance was explained by a single factor and the instrument was able to differentiate their scores as a function of nutritional status $(p<0.05)$, and correlate with measures of body dissatisfaction $(r h o=0.50)$. Confirmed its internal consistency $(\alpha>0.88)$, no difference between scores (test-retest) $(p<0.68)$ and introducing the coefficient of intra-class correlation of 0.93 . It was concluded that the EAT-26 demonstrated its psychometric qualities for the group studied.
\end{abstract}

Keywords: validity, reproducibility, psychometry, eating disorders, adolescents

O crescimento do interesse na investigação dos fatores relacionados aos transtornos alimentares (TAs) tem se refletido no aumento do número de instrumentos destinados a avaliar seus sintomas e fatores de risco. Entre os TAs mais comuns estão a Anorexia Nervosa (AN), caracterizada pela recusa do indivíduo a manter um peso corporal na faixa normal mínima e um temor intenso de ganhar peso (American Psychiatric Association, 2013); e a Bulimia Nervosa (BN), cujas características essenciais consistem de compulsões periódicas e métodos compensatórios inadequados para evitar o ganho de peso (DSM-IV-TR, 2002). Além disso, ambas as manifestações são marcadas por uma preocupação excessiva com o peso e a forma corporal (Souza, Souza, Hirai, Luciano, \& Souza, 2011).

Em relação à prevalência desses transtornos, estima-se que seja de $1 \%$ a $5 \%$ entre a população geral, sendo mais comum em mulheres (Alves, Vasconcelos, Calvo, \& Neves, 2008; Souza \& Santos, 2012). No entanto, nos últimos

1 Endereço para correspondência: Rua Clóvis Beviláqua, 163/1003, Madalena, Recife, PE. Brasil. CEP: 50.710-330. E-mail: leodesousafortes@hotmail.com anos, sua manifestação tem sido relatada, também, entre a população masculina, numa proporção de 10 para 1 caso em relação às mulheres (American Psychiatric Association, 2000). Mesmo assim, sua etiologia para essa população ainda não é clara, necessitando, portanto, de maiores investigações. Nesse contexto, os pesquisadores necessitam de instrumentos com atestadas propriedades psicométricas para investigação dos fatores relacionados a estes transtornos (Thompson, 2004).

No cenário clínico e de pesquisa, o Eating Atitudes Test (EAT) é um dos instrumentos mais aplicados para o rastreamento de sintomas e comportamentos de risco para o desenvolvimento de TAs (Türy, Guleç, \& Kohls, 2010). Sua primeira versão, desenvolvida por Garner et al. (1979), foi composta por 40 itens, em escala Likert de pontos, de 1 (sempre) a 6 (nunca). No entanto, em função do tempo gasto em seu preenchimento, uma versão abreviada foi proposta (Garner, Olmsted, Bohr, \& Garfinkel,, 1982), com 26 itens. Essa versão simplificada foi estruturada em três fatores: 1) dieta - reflete restrição dietética para alimentos com altos valores calóricos, evitação de comida e preocupação 
excessiva com magreza; 2) bulimia e preocupação com alimentos - diz respeito a episódios de ingestão exagerada de alimentos, seguidos por métodos patológicos de controle de peso corporal; 3) autocontrole oral - representa o autocontrole em relação à comida e avalia a influência que o ambiente pode exercer sobre os hábitos alimentares.

O EAT-26 já foi traduzido e validado para diversas populações (Bighetti, Santos, Santos, \& Ribeiro, 2004; Garner et al., 1982; Lane, Lane, \& Matheson, 2004; Rivas, Bersabé, Jumenez, \& Berrocal, 2010), sendo que poucas delas foram compostas por amostras masculinas (Lane et al., 2004). No Brasil, o EAT-26 teve suas qualidades psicométricas avaliadas para adolescentes do sexo feminino (Bighetti et al., 2004), mas esse cuidado ainda não foi dado aos adolescentes brasileiros do sexo masculino. Apesar disso, alguns estudos nacionais já utilizaram o EAT-26 em amostras compostas por meninos (Chiodini \& Oliveira, 2003; Fortes \& Ferreira, 2011; Vieira et al., 2006).

Diante do exposto, e considerando a importância de se investigar a etiologia dos TAs também entre meninos, o presente estudo teve como objetivo avaliar as propriedades psicométricas do EAT-26 para os adolescentes do sexo masculino.

\section{Método}

\section{Participantes}

Trata-se de um estudo transversal realizado com adolescentes do sexo masculino com idades entre 10 e 19 anos da cidade de Juiz de Fora/MG. Segundo a Secretaria de Educação de Juiz de Fora, essa população era na ordem de 50.000 sujeitos no ano de 2011. Por conseguinte, realizouse cálculo amostral, considerando os seguintes critérios: prevalência de $30 \%$ para comportamentos alimentares inadequados (Alves et al., 2008), 95\% de confiança, 5\% de erro amostral e 1,4 para efeito de desenho, totalizando 322 sujeitos que deveriam ser avaliados para compor amostra representativa da população. Em adição, Reichenheim e Moraes (2007) recomendam avaliar 10 sujeitos para cada item do questionário em investigações de validação psicométrica de instrumentos. O EAT-26 apresenta 26 questões. Sendo assim, seria necessário o recrutamento mínimo de 260 jovens. No entanto, foram avaliados 403 adolescentes, dos quais 46 foram excluídos da pesquisa por não responderem os questionários em sua totalidade ou por não participarem das aferições antropométricas.

A amostra proporcional foi estratificada segundo a inserção das escolas nas regiões sócio-geográficas do município de Juiz de Fora (norte, sul e centro) e o tipo de vinculação administrativa (pública e privada) e, em seguida, distribuída no ensino fundamental e médio.

A seleção ocorreu aleatoriamente, por meio de sorteio simples, em duas etapas. Realizou-se, primeiro, o sorteio das escolas em cada região e, posteriormente, o sorteio dos adolescentes nessas unidades. As escolas foram selecionadas valendo-se da relação fornecida pelo setor de estatística da Secretaria de Educação do Estado de Minas Gerais. A amostra final da pesquisa foi distribuída em seis pontos diferentes de coleta (escolas) e constituída por adolescentes presentes nas escolas nos dias da coleta.

Foram incluídos no estudo somente os jovens regularmente matriculados em escolas privadas ou públicas de Juiz de Fora/ MG (190 matriculados em instituições privadas e 167, em públicas), que apresentassem o Termo de Consentimento Livre e Esclarecido (TCLE) assinado pelo responsável, além de ter disponibilidade para responder questionários e participar de medidas antropométricas. Acrescentando, os participantes também deveriam assinar o TCLE para participar do estudo.

O projeto obteve aprovação do Comitê de Ética e Pesquisa em Seres Humanos da Universidade Federal de Juiz de Fora (protocolo 2282.022.2011 - parecer 022/2011), de acordo com a lei 196/96 do Conselho Nacional de Saúde.

\section{Instrumentos}

Eating Attitudes Test (EAT-26). O EAT-26 (Garner et al., 1982) é um questionário de autopreenchimento, composto por 26 questões na forma de escala Likert de pontos (sempre $=3$; muitas vezes $=2$; frequentemente $=1$; poucas vezes, quase nunca e nunca $=0$ ). A questão 25 apresenta pontuação invertida, ou seja, as alternativas sempre, muitas vezes e frequentemente são avaliadas com peso 0 , a resposta poucas vezes apresenta peso 1 , quase nunca peso 2 e nunca valor 3 . $\mathrm{O}$ escore é calculado a partir da soma das respostas de cada item, variando de 0 a 78 pontos, sendo que quanto maior o escore, maior o risco de desenvolvimento de TA. Considera-se que escores maiores que 21 são indicativos de comportamento alimentar de risco para TAs (Garner et al., 1982; Rivas et al., 2010). O questionário é composto por três subescalas, cada qual avaliando fatores distintos do comportamento alimentar: dieta (13 itens), bulimia e preocupação com alimentos (seis itens) e autocontrole oral (sete itens).

A versão utilizada neste estudo teve sua equivalência semântica para a língua portuguesa avaliada e descrita por Bighetti et al. (2004). Neste estudo, os autores ainda comprovaram a consistência interna satisfatória do instrumento entre uma amostra de meninas adolescentes.

Body Shape Questionnaire (BSQ). O BSQ é um questionário de autopreenchimento, composto por 34 perguntas destinadas a avaliar a preocupação e a atitude com a forma e peso corporais nas últimas quatro semanas. O escore total é calculado por meio da soma das respostas, variando de 34 a 204 pontos, sendo que quanto maior a pontuação, maior a insatisfação corporal. Essa escala possui uma versão brasileira validada para adolescentes de ambos os sexos (Conti, Cordás, \& Latorre, 2009) e sua análise de consistência interna revelou um $\alpha=0,96$ entre meninos e meninas e um coeficiente de correlação entre os escores do teste-reteste significativo, variando de 0,89 a 0,91 para meninas e meninos, respectivamente.

Considerando que um dos principais fatores de risco para o desenvolvimento dos TAs é a insatisfação corporal (American Psychiatric Association, 2000), espera-se uma associação entre os escores do EAT-26 e do BSQ, sendo este um critério aplicado para a avaliação da validade concorrente. 
Avaliação antropométrica. A avaliação antropométrica baseou-se na aferição de massa corporal e estatura dos participantes. Os procedimentos para as coletas dessas variáveis foram realizados de acordo com as padronizações determinadas pela ISAK (2001). A massa corporal foi mensurada por uma balança digital portátil da marca Tanita com precisão de $100 \mathrm{~g}$ e capacidade máxima de $200 \mathrm{~kg}$. Foi utilizado estadiômetro portátil com precisão de $0,1 \mathrm{~cm}$ e altura máxima de 2,20m da marca Welmy para mensurar a estatura dos adolescentes.

O indicador utilizado para avaliação do estado nutricional foi o índice de massa corporal/idade (IMC/I). A classificação seguiu as recomendações da World Health Organization (2007): se IMC/I apresenta-se menor que o percentil $5^{\circ}$, o adolescente é classificado com baixo peso; entre percentil $5^{\circ}$ e percentil $85^{\circ}$, peso normal; entre percentil $85^{\circ}$ e percentil $95^{\circ}$, sobrepeso; e sujeitos com IMC/I maior que percentil $95^{\circ}$ são classificados como obesos.

Neste estudo, a utilização dos dados antropométricos (IMC) teve a função de comprovar a validade discriminante do EAT-26, ou seja, avaliar sua capacidade em discriminar diferentes grupos de estado nutricional. Esse procedimento baseia-se nos dados clínicos e populacionais que descrevem adolescentes apresentando correlação positiva entre IMC e comportamento alimentar inadequado (Alves et al., 2008; Martins, Pelegrini, Matheus, \& Petroski, 2010).

\section{Procedimentos}

Os alunos selecionados aleatoriamente com idade entre 10 e 19 anos, que estavam presentes no dia da coleta, foram convidados a participar do estudo. A aplicação dos questionários deu-se na forma de autopreenchimento, contendo os dois instrumentos (EAT-26 e BSQ). Foi conduzida em grupos, dentro das próprias salas de aula e no período escolar, após autorização do professor responsável pela turma.

A avaliação antropométrica foi realizada individualmente em uma sala disponibilizada pela instituição de ensino, logo após a aplicação dos questionários. A massa corporal e a estatura foram aferidas por um mesmo pesquisador, que zelou pela padronização das medidas. Selecionou-se, aleatoriamente, três turmas para a realização da análise de reprodutibilidade ( $\mathrm{n}=49)$, com a aplicação do EAT-26 em dois pontos no tempo, com intervalo de duas semanas entre as aplicações (Reichenheim \& Moraes, 2007).

Alguns cuidados foram tomados, apoiando-se nos padrões vigentes de estudo de validade (Reichenheim \& Moraes, 2007). Deu-se especial atenção ao procedimento da coleta, com o intuito de evitar vieses no registro da informação. Os dados foram coletados por um único pesquisador, garantindose, assim, a padronização das orientações e esclarecimentos de dúvidas.

\section{Análise Estatística}

Todos os dados foram analisados, em um primeiro momento, por medidas de tendência central (médias, desvios-padrão, medianas, valores mínimos e máximos). Para verificação da validade de construto, o instrumento foi submetido a uma análise fatorial exploratória, utilizando o método de componentes principais para extração dos fatores. Conduziu-se a rotação oblimin, conforme o artigo de validação original do EAT-26 (Gardner et al., 1982). A validade discriminante foi avaliada mediante comparação dos escores do EAT-26 segundo as classificações do estado nutricional, por meio do modelo de análise de variância de um caminho (ANOVA oneway). O post hoc de Bonferroni foi utilizado para identificar entre quais subgrupos do estado nutricional estavam as diferenças. Para a validade concorrente utilizou-se a análise de correlação de Spearman Rank entre os escores do BSQ e EAT-26. A consistência interna foi avaliada por meio do coeficiente alfa de Cronbach. Para a avaliação da reprodutibilidade foi realizada a comparação dos escores entre os momentos 1 e 2 (teste de Wilcoxon) e também foi calculado o coeficiente de correlação intra-classe $\left(r_{i c c}\right)$. Foram utilizados testes paramétricos quando as suposições de normalidade e homogeneidade de variâncias foram atendidas e testes não paramétricos quando estas suposições foram violadas. A análise estatística foi realizada no programa SPSS versão 17.0. Em todas as análises considerou-se o nível de significância $5 \%$.

\section{Resultados}

A análise descritiva das variáveis idade, IMC e os escores dos participantes para o EAT-26 e para o BSQ estão descritos na Tabela 1.

Tabela 1. Análise Descritiva das variáveis idade, IMC, EAT-26 e $B S Q$

\begin{tabular}{llllll}
\hline Variável & Média & DP & Mediana & Mínimo & Máximo \\
\hline Idade (anos) & 14,2 & 2,2 & 14,0 & 10,0 & 19,0 \\
IMC (Kg/m2) & 20,8 & 3,3 & 20,4 & 14,0 & 33,1 \\
Escore EAT-26 & 12,6 & 12,6 & 9,0 & 0 & 73,0 \\
Escore BSQ & 62,4 & 24,3 & 56,0 & 13,0 & 178,0 \\
\hline Nota. O Eating Attitudes Test (EAT-26) foi retirado de Bigheti et al.
\end{tabular}

(2004); o Body Shape Questionnaire (BSQ) foi retirado de Conti et al. (2010).

No que concerne aos escores do EAT-26, os achados indicaram que 62 jovens $(17,4 \%)$ tinham riscos para os TAs (pontuação maior ou igual a 21).

\section{Análise Fatorial Exploratória e Consistência Interna}

A medida de adequação KMO (Kaiser-Meyer-Olkin measure of sampling adequacy) foi de 0,92 e o teste de esfericidade de Bartlett teve valor de $3567(p<0,01)$, indicando que a amostra do estudo foi adequada para a realização da análise fatorial. Um único fator foi responsável por explicar 32,8\% da variância total dos dados. A Tabela 2 apresenta os resultados da análise de fatores. A maioria dos itens apresentou cargas fatoriais acima de 0,30 , exceto os itens 19 e 25. Estes dois itens também apresentaram valores 
de correlação com a escala total abaixo de 0,30. O alfa de Cronbach para a escala foi de 0,88 e apresentaria um sutil aumento se os itens 19 e 25 fossem retirados $(\alpha=0,90)$. O EAT-26 apresentou uma estrutura unidimensional com uma alta consistência interna, mesmo com os dois itens que mereceram revisão.

\section{Validade Discriminante e Concorrente}

$\mathrm{Na}$ validação discriminante, houve diferença estatisticamente significativa $(p<0,05)$ nas médias dos escores entre os quatro subgrupos de estado nutricional (Tabela 3). Na análise da validação concorrente, foi registrada correlação estatisticamente significativa entre os escores do EAT-26 e BSQ (Tabela 3).

Tabela 2. Estrutura Fatorial e Análise dos Itens da Versão Masculina do EAT-26

\begin{tabular}{|c|c|c|c|c|}
\hline \multirow[b]{2}{*}{ Item } & \multicolumn{2}{|c|}{ Estrutura Fatorial } & \multicolumn{2}{|c|}{ Análise dos Itens } \\
\hline & Eigenvalue & $\begin{array}{l}\text { Carga } \\
\text { fatorial }\end{array}$ & Correlaçãa $^{1}$ & $\begin{array}{c}\alpha \text { se o item } \\
\text { for excluído }\end{array}$ \\
\hline 1 & 8,53 & 0,49 & 0,46 & 0,87 \\
\hline 2 & 2,04 & 0,70 & 0,62 & 0,87 \\
\hline 3 & 1,44 & 0,54 & 0,51 & 0,87 \\
\hline 4 & 1,23 & 0,54 & 0,45 & 0,87 \\
\hline 5 & 1,08 & 0,72 & 0,66 & 0,87 \\
\hline 6 & 0,98 & 0,65 & 0,60 & 0,87 \\
\hline 7 & 0,93 & 0,73 & 0,66 & 0,87 \\
\hline 8 & 0,85 & 0,35 & 0,30 & 0,88 \\
\hline 9 & 0,75 & 0,73 & 0,64 & 0,87 \\
\hline 10 & 0,73 & 0,72 & 0,65 & 0,87 \\
\hline 11 & 0,68 & 0,64 & 0,58 & 0,87 \\
\hline 12 & 0,66 & 0,43 & 0,39 & 0,88 \\
\hline 13 & 0,64 & 0,36 & 0,33 & 0,88 \\
\hline 14 & 0,59 & 0,54 & 0,49 & 0,87 \\
\hline 15 & 0,57 & 0,40 & 0,38 & 0,88 \\
\hline 16 & 0,56 & 0,67 & 0,60 & 0,87 \\
\hline 17 & 0,54 & 0,66 & 0,60 & 0,87 \\
\hline 18 & 0,46 & 0,51 & 0,45 & 0,87 \\
\hline 19 & 0,44 & 0,26 & 0,24 & 0,89 \\
\hline 20 & 0,42 & 0,34 & 0,31 & 0,88 \\
\hline 21 & 0,40 & 0,46 & 0,37 & 0,88 \\
\hline 22 & 0,36 & 0,70 & 0,62 & 0,87 \\
\hline 23 & 0,31 & 0,73 & 0,66 & 0,87 \\
\hline 24 & 0,30 & 0,56 & 0,49 & 0,87 \\
\hline 25 & 0,27 & $-0,13$ & $-0,14$ & 0,89 \\
\hline \multirow[t]{2}{*}{26} & 0,26 & 0,72 & 0,63 & 0,87 \\
\hline & \multicolumn{2}{|c|}{$\begin{array}{c}\text { \% variância explicada } \\
=32,79\end{array}$} & \multicolumn{2}{|c|}{$\alpha=0,88$} \\
\hline
\end{tabular}

Nota. ${ }^{1}$ Correlação entre o item e o total da escala; $\alpha=$ alfa de Cronbach

\section{Reprodutibilidade}

Não houve diferença estatisticamente significativa nas médias dos escores do EAT-26, quando foram considerados o momento 1 (teste) e o momento 2 (reteste). O coeficiente de correlação intra-classe foi significativo com magnitude de $0,93(p<0,01)$ (Tabela 3$)$.

\section{Discussão}

Estudos para avaliação de propriedades psicométricas de um instrumento são essenciais antes de sua aplicação em um contexto cultural diferente do qual foi criado (Cash, 2004; Thompson, 2004). Os resultados do presente estudo foram satisfatórios, o que permite inferir que o EAT-26 apresenta bons indícios psicométricos para sua utilização entre adolescentes do sexo masculino.

$\mathrm{Na}$ análise fatorial exploratória, a melhor estrutura obtida foi composta por um único fator, que explicou 32,8\% da variância total dos dados. Os itens 19 ("Demonstro autocontrole diante dos alimentos") e 25 ("Gosto de experimentar novos alimentos ricos em calorias") apresentaram cargas fatoriais baixas e baixa correlação com o total da escala, o que pode estar sinalizando falta de entendimento nessas questões, aspecto esse a ser revisto e observado em outros estudos com este público. Mesmo assim, o EAT-26 apresentou excelente consistência interna $(\alpha=0,88)$, incluindo os dois itens pouco homogêneos.

A estrutura unidimensional encontrada, a porcentagem da variância explicada pela análise fatorial $(32,8 \%)$ e a consistência interna do EAT-26 são muito semelhantes aos resultados encontrados na validação espanhola do instrumento, realizada com duas amostras de mulheres, uma composta de estudantes de escolas públicas e privadas $(36,8 \%$ da variância explicada e $\alpha=0,90$ ) e a outra composta de pessoas com distúrbios alimentares pareadas segundo o sexo e a idade com pessoas sem o distúrbio (46,5\% da variância explicada e $\alpha=0,93$ ) (Rivas et al., 2010). Nesse sentido, os resultados obtidos são consistentes com achados anteriores e também sugerem que os itens sejam agrupados em uma única dimensão. Embora no estudo original de validade e reprodutibilidade do EAT-26 seja apresentada uma estrutura de três fatores, os próprios autores discutem que essa estrutura tem que ser vista com cautela, uma vez que não havia um número suficiente de sujeitos para que a análise fosse mais consistente (Garner et al., 1982).

Em relação à versão do EAT-26 validada para adolescentes brasileiras do sexo feminino, Bighetti (2003) demonstrou extração de três fatores (Dieta; Bulimia e Preocupação com Alimentos; Autocontrole Oral) pela análise de componentes principais, conforme também foi evidenciado no estudo original desse instrumento (Garner et al., 1982). No entanto, devido às cargas fatoriais encontradas na pesquisa de Bighetti, alguns itens se alocaram diferentemente. Por exemplo, as questões 2, 3 e 5 foram incluídas na subescala Dieta. Do mesmo modo, os itens 1, 10, 11, 19 e 24 foram adicionados a subescala Bulimia e Preocupação com Alimentos. Sendo assim, a estrutura fatorial do EAT-26 para jovens brasileiras do sexo feminino, assim como na presente investigação 
Tabela 3. Valores Referentes à Avaliação Psicométrica do EAT-26 para Adolescentes Brasileiros do Sexo Masculino

\begin{tabular}{|c|c|c|c|c|c|}
\hline Análise & Variável & Parâmetro & Categoria & & $\begin{array}{l}\text { valor } \\
\text { de } p\end{array}$ \\
\hline \multirow[t]{4}{*}{ Validade Discriminante } & Escore EAT-26 - Estado Nutricional & $\mathrm{M}( \pm \mathrm{DP})$ & Baixo Peso & $10,55( \pm 3.95) b, d$ & $<0,05$ \\
\hline & & & Peso normal & $13,42( \pm 1,29) \mathrm{d}$ & \\
\hline & & & Sobrepeso & $14,83( \pm 2,65) \mathrm{d}$ & \\
\hline & & & Obeso & $21,07( \pm 4,23)$ & \\
\hline Validade Concorrente & Escore EAT-26 - BSQ & rspearman & & 0,50 & $<0,01$ \\
\hline Reprodutibilidade & Escore EAT-26 & Diferença de Médias & & 0,76 & 0,10 \\
\hline$(n=49)$ & T1 vs T2 & $\mathrm{r}_{\mathrm{icc}}$ & & 0,93 & $<0,01$ \\
\hline
\end{tabular}

(jovens brasileiros do sexo masculino), apresentou-se diferentemente quando comparada ao instrumento original (Garner et al., 1982). Todavia, decidiu-se manter todas as questões do EAT-26, pois segundo Reichenheim e Moraes (2007), a busca pelo máximo de equivalência entre o questionário original e o traduzido deve guiar todo o processo de maneira a evitar formas, muitas vezes sutis, de distorção. Atenção ainda maior deve ser dada quando o fenômeno a ser avaliado trata-se de aspecto subjetivo, como são os fenômenos psicopatológicos.

Ademais, o EAT-26 da presente pesquisa foi capaz de diferenciar adolescentes de acordo com o seu estado nutricional. Sujeitos classificados como "obesos" apresentaram maiores escores de hábitos alimentares inadequados quando comparados a indivíduos com "peso normal" e "baixo peso". No estudo original (Garner et al., 1982), a validade discriminante foi realizada mediante comparação das pontuações do EAT-26 entre mulheres com diagnóstico clínico para AN e grupo controle. Entretanto, pode-se afirmar que essa distinção entre os adolescentes em diferentes estágios nutricionais também é relevante, visto o consenso de que o adolescente com sobrepeso/obesidade tende a apresentar maior risco para TAs, por tenderem a fazer dietas restritivas com mais frequência (Bernardi, Harb, Levandovski, \& Hidalgo, 2009; Scherer, Martins, Pelegrini, Matheus, \& Petroski, 2010). Além disso, outros estudos têm utilizado o mesmo critério para a validade discriminante entre amostras brasileiras (Conti, Cordás et al., 2009; Conti, Slater, \& Latorre, 2009).

Os escores do EAT-26 correlacionaram-se com os obtidos no BSQ. A insatisfação corporal é, comprovadamente, um dos aspectos principais para o diagnóstico dos transtornos alimentares (American Psychiatric Association, 2000). Outros estudos identificaram resultados semelhantes em relação à associação de comportamentos alimentares de risco e insatisfação corporal, mesmo com a aplicação de outros instrumentos (Garner et al., 1982; Rivas et al., 2010). A utilização do Body Shape Questionnaire apoiou-se no fato de ser um dos questionários mais utilizados para avaliação da satisfação corporal (Conti, Cordás et al., 2009; Magalhães
\& Mendonça, 2005) e sua validade para a população adolescente masculina brasileira já ter sido comprovada (Conti, Cordás et al., 2009). Dessa forma, a obtenção de correlação significativa entre esses instrumentos comprova a validade concorrente do EAT-26 entre a amostra estudada.

Os resultados evidenciaram, ainda, que o EAT-26 manteve-se estável entre os dois momentos, apresentando boa reprodutibilidade. Não se identificou diferença estatisticamente significativa entre os escores obtidos nos momentos 1 e 2 . Outros estudos de validade do EAT-26 não deram a atenção a essa análise (Bighetti et al., 2004; Garner et al., 1982; Rivas et al. 2010), apesar de sua recomendação por especialistas da área (Reicheheim \& Moraes, 2007; Thompson, 2004).

Obteve-se média de 12,65 para o escore do EAT-26 entre os adolescentes estudados. Entretanto, a análise desse resultado à luz da literatura é dificultada em função de os outros estudos de validade do EAT-26 utilizarem apenas amostras do sexo feminino (Bighetti et al., 2004; Garner et al., 1982; Martins et al., 2010; Nunes, Camey, Olinto, \& Mari, 2005; Rivas et al., 2010; Scherer et al., 2010). Por fim, os resultados da presente investigação indicaram $17,4 \%$ para prevalência de risco concernente aos TAs. Sendo assim, esses achados corroboram outras pesquisas (Fortes \& Ferreira, 2011; Scherer et al., 2010), os quais identificaram prevalência de aproximadamente $20 \%$ para escores maiores ou iguais a 21 no EAT-26.

O EAT-26, além de ser um importante instrumento, é um dos mais aplicados no rastreamento de comportamentos alimentares de risco para os TAs (Bighetti et al., 2004; Rivas et al., 2010). Grande parte dos estudos com enfoque no desenvolvimento infanto-juvenil tem investigado hábitos alimentares inapropriados, associando-os a aspectos como idade, sexo (Martins et al., 2010), estado nutricional (Scherer et al., 2010), qualidade da relação familiar (Rossi, Moreira, \& Rauen, 2008), pressão social (Rivas et al., 2010) e insatisfação corporal (Fortes \& Ferreira, 2011). Entretanto, muitos deles não utilizaram instrumentos com comprovadas qualidades psicométricas (Martins et al., 2010; Scherer et al., 2010; Vieira et al., 2006). 
Entre as limitações deste estudo pode-se destacar o fato de não ter sido utilizada amostra clínica para avaliação da validade discriminante, como no estudo original (Garner et al., 1982). Justifica-se, entretanto, a dificuldade de acesso a grupos de adolescentes do sexo masculino com diagnóstico clínico para AN e/ou BN. Além disso, pode-se destacar a obtenção de uma estrutura fatorial única, distinta da encontrada no estudo original de validade, porém sustentada por outras pesquisas que mostram a complexidade deste instrumento, especialmente para o sexo masculino.

O EAT-26 comprovou suas propriedades psicométricas para o grupo estudado. Além de estrutura fatorial satisfatória, o instrumento correlacionou-se com as medidas de avaliação da insatisfação corporal e discriminou diferentes estratos de IMC, como também comprovou sua consistência interna e reprodutibilidade. Mais estudos são necessários no sentido de confirmar sua estrutura fatorial entre outras amostras, a fim de confirmar sua validade externa.

\section{Referências}

American Psychiatric Association. (2000). Appendix I: Outline for cultural formulation and glossary of culturebound syndromes. Diagnostic and Statistical Manual of Mental Disorders. doi: http://dx.doi.org 10.1176/appi.books.9780890423349.7060

American Psychiatry Association (2013). Feeding and eating disorders. Diagnostic and Statistical Manual of Mental Disorders, Fifth Edition, Edited by American Psychiatric Association. doi: 10.1176/appi.books.9780890425596.dsm10

Alves, E., Vasconcelos, F. A. G., Calvo, M. C. M., \& Neves, J. (2008). Prevalência de sintomas de anorexia nervosa e insatisfação com a imagem corporal em adolescentes do sexo feminino do Município de Florianópolis, Santa Catarina, Brasil. Cadernos de Saúde Pública, 24, 503-512. doi: http://dx.doi. org/10.1590/S0102-311X2008000300004

Bernardi, F., Harb, A. B. C., Levandovski, R. M., \& Hidalgo, M. P. L. (2009). Transtornos alimentares e padrão circadiano alimentar: Uma revisão. Revista de Psiquiatria do Rio Grande do Sul, 31, 170-176. doi: http://dx.doi.org/10.1590/S010181082009000300006

Bigheti, F. (2003). Tradução e validação do Eating Attitudes Test (EAT-26) em adolescentes do sexo feminino na cidade de Ribeirão Preto-SP (Unpublished master's thesis). Universidade de São Paulo, São Paulo.

Bigheti, F., Santos, C. B., Santos, J. E., \& Ribeiro, R. P. P. (2004). Tradução e avaliação do Eating Attitudes Test em adolescentes do sexo feminino de Ribeirão Preto, São Paulo. Jornal Brasileiro de Psiquiatria, 53(6), 339-346.

Cash, T. (2004). Body image: past, present, and future. Body Image, 1, 1-5. doi: http://dx.doi.org/10.1016/S1740-1445(03)00011-1

Chiodini, J. S., \& Oliveira, M. R. M. (2003). Comportamento Alimentar de Adolescentes - Aplicação do EAT-26 em uma escola pública. Saúde em Revista, 5, 53-58.

Conti, M. A., Cordás, T. A., \& Latorre, M. R. D. O. (2009). Estudo de validade e confiabilidade da versão brasileira do Body Shape Questionnaire (BSQ) para adolescentes. Revista Brasileira de Saúde Materna e Infantil, 9, 331-338. doi: http://dx.doi. org/10.1590/S1519-38292009000300012
Conti, M. A., Slater, B., \& Latorre, M. R. D. O. (2009). Validação e reprodutibilidade da Escala de Evaluación de Insatisfación Corporal para Adolescentes. Revista de Saúde Pública, 43, 515-524. doi: http://dx.doi.org/10.1590/S003489102009000300016

Fortes, L. S., \& Ferreira, M. E. C. (2011). Comparação da insatisfação corporal e do comportamento alimentar inadequado em atletas adolescentes de diferentes modalidades esportivas. Revista Brasileira de Educação Física e Esporte, 25, 707-716. doi: http://dx.doi.org/10.1590/S1807-55092011000400014

Garner, D. M., Olmsted, M. P., Bohr, Y., \& Garfinkel, P. A. (1982). The eating attitudes test: psychometric features and clinical correlations. Psychology Medicine, 12, 871-878.

ISAK. (2001). The International Society for Advancement for Kineanthropometry (1a. ed.). Australia: National Library of Australia.

Lane, H. J., Lane, A. M., \& Matheson, H. (2004). Validity of Eating Atitude Test among exercisers. Journal of Sport Science and Medicine, 3, 244-253.

Magalhães, V. C., \& Mendonça, G. A. S. (2005). Transtornos alimentares em universitárias: Estudo de confiabilidade da versão brasileira de questionários autopreenchíveis. Revista Brasileira de Epidemiologia, 8, 236-245. doi: http://dx.doi. org/10.1590/S1415-790X2005000300005

Martins, C. R., Pelegrini, A., Matheus, S. C., \& Petroski, E. L. (2010). Insatisfação com a imagem corporal e a relação com estado nutricional, adiposidade corporal e sintomas de anorexia e bulimia em adolescentes. Revista de Psiquiatria do Rio Grande do Sul, 32, 19-23. doi: http://dx.doi.org/10.1590/ S0101-81082010000100004

Nunes, M. A., Camey, S., Olinto, M. T. A., \& Mari, J. J. (2005). The validity and 4-year test-retest reliability of the Brazilian version of the Eating Attitudes Test-26. Brazilian Journal of Medical and Biological Research, 38, 1655-1662. doi: http:// dx.doi.org/10.1590/S0100-879X2005001100013

Reichenheim, M. E., \& Moraes, C. L. (2007). Operacionalização de adaptação transcultural de instrumentos de aferição usados em epidemiologia. Revista de Saúde Pública, 41, 665-73. doi: http://dx.doi.org/10.1590/S0034-89102006005000035

Rivas, T., Bersabé, R., Jumenez, M., \& Berrocal, C. (2010). The eating attitudes test (EAT-26) - Reliability and validity in Spanish female samples. The Spanish Journal Psychology, 13, 1044-1056.

Rossi, A., Moreira, E. A. M., \& Rauen, M. S. (2008). Determinantes do comportamento alimentar: uma revisão com enfoque na família. Revista de Nutrição, 21, 739-748. doi: http://dx.doi. org/10.1590/S1415-52732008000600012

Scherer, F. C., Martins, C. R., Pelegrini, A., Matheus, S. C., \& Petroski, E. L. (2010). Imagem corporal em adolescentes: Associação com a maturação sexual e sintomas de transtornos alimentares. Jornal Brasileiro de Psiquiatria, 59, 198-202. doi: http://dx.doi.org/10.1590/S0047-20852010000300005

Souza, A. A., Souza, J. C., Hirai, E. S., Luciano, H. A., \& Souza, N. (2011). Estudo sobre a anorexia e bulimia nervosa em universitárias. Psicologia: Teoria e Pesquisa, 27, 195-198. doi: http://dx.doi.org/10.1590/S0102-37722011000200012

Souza, L. V., \& Santos, M. A. (2012). Familiares de pessoas diagnosticadas com transtornos alimentares: Participação em atendimento grupal. Psicologia: Teoria e Pesquisa, 28, 325-334. doi: http://dx.doi.org/10.1590/S0102-37722012000300008 
Thompson, J. K. (2004). The (mis)measurement of body image: Ten strategies to improve assessment for applied and research purposes. Body Image, 1, 7-14. doi: :

Türy, F., Guleç, H., \& Kohls, E. (2010). Assessment methods for eating disorders and body image disorders. Journal of Psychosomatic Research, 69, 601-611. doi: http://dx.doi. org/10.1016/j.jpsychores.2009.05.012

Vieira, L. F., Oliveira, L. P., Viveira, J. L., Vissoci, J. R. N., Hoshino, E. F., \& Fernandes, S. L. (2006). Distúrbios de atitudes alimentares e sua relação com a distorção da auto-imagem corporal em atletas de judô do estado do Paraná. Revista da Educação Física/UEM, 17, 177-184.
World Health Organization. (2007). Development of a WHO growth reference for school-aged children and adolescents. Bulletin of the World Health Organization, 85, 660-667. doi: http://dx.doi. org/10.2471/BLT.07.04349

Recebido em 30.01.2013

Primeira decisão editorial em 28.02.2016

Versão final em 10.03.2016

Aceito em 25.04.2016 